\title{
Basics of a generalized Wiman-Valiron theory for monogenic Taylor series of finite convergence radius
}

\author{
D. Constales ${ }^{1 \star}$, R. De Almeida ${ }^{2 \star \star}$, R.S. Kraußhar ${ }^{3}$ \\ 1 Department of Mathematical Analysis, Ghent University, Building S-22, Gal- \\ glaan 2, B-9000 Ghent, Belgium \\ 2 Departamento de Matemática, Universidade de Trás-os-Montes e Alto Douro, \\ P-5000-911 Vila Real, Portugal. \\ 3 Department of Mathematics, Katholieke Universiteit Leuven, Celestijnenlaan \\ 200-B, B-3001 Heverlee, Belgium.
}

The date of receipt and acceptance will be inserted by the editor - (c) SpringerVerlag 2007

\begin{abstract}
In this paper we develop the basic concepts for a generalized Wiman-Valiron theory for Clifford algebra valued functions that satisfy inside an $n+1$-dimensional ball the higher dimensional Cauchy-Riemann system $\frac{\partial f}{\partial x_{0}}+\sum_{i=1}^{n} e_{i} \frac{\partial f}{\partial x_{i}}=0$. These functions are called monogenic or Clifford holomorphic inside the ball. We introduce growth orders, the maximum term and a generalization of the central index for monogenic Taylor series of finite convergence radius. Our goal is to establish explicit relations between these entities in order to estimate the asymptotic growth behavior of a monogenic function in a ball in terms of its Taylor coefficients. Furthermore, we exhibit a relation between the growth order of such a function $f$ and the growth order of its partial derivatives.
\end{abstract}

Mathematics Subject Classification (2000): 30G35

\section{Introduction}

The study of the asymptotic growth behavior of holomorphic and meromorphic functions belongs to one of the classical topics of complex analysis.

* Financial support from BOF/GOA 01GA0405 of Ghent University gratefully acknowledged.

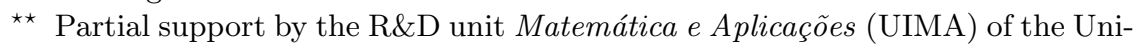
versity of Aveiro, through the Portuguese Foundation for Science and Technology $(\mathrm{FCT})$ 
Early contributions in this direction came from E. Lindelöf [15] and A. Pringsheim [18] and a short time later by the school of A. Wiman [22] and G. Valiron [21] in the first two decades of the 20th century. With the notion of growth orders, the maximum term of a Taylor series and the central index they performed a quantitative asymptotic analysis. Their results addressed exclusively the context of entire holomorphic functions.

It appeared to be a difficult task to develop a more general theory for functions that are not entire holomorphic.

Breakthrough works in that direction were provided by R. Nevanlinna [17] and his students in 1925 who managed to build up a quantitative description of the growth behavior of all functions that are meromorphic in the entire complex plane. For a compact description we refer for instance to $[13,14]$.

In $[19,20]$ one finds a description of a properly adapted version of WimanValiron theory to the concrete case of functions in one complex variable that are holomorphic in a ball of finite radius. Already in the one complex variable case, the relation between the growth orders, the maximum term and the central index of the Taylor series turned out to be much more complicated than in the entire case. In many situations one only obtains estimates in one direction between these entities for which one has the equality relation in the entire holomorphic case. See for instance $[16,20]$. In several complex variables the treatment of analogous questions gets even more complicated due to the lack of a direct analogue of Hadamard's formula which relates the Taylor coefficients to the radius of convergence in the one complex variable case.

In this paper we study analogous questions within the context of Clifford algebra valued functions defined in open subsets of $\mathbb{R}^{n+1}$ that are solutions to the higher dimensional Cauchy-Riemann system $D f=0$ where $D:=\frac{\partial}{\partial x_{0}}+\sum_{i=1}^{n} \frac{\partial}{\partial x_{i}} e_{i}$. Solutions to this system are often called monogenic or Clifford holomorphic. Many classical theorems from complex analysis, such as for instance the Cauchy integral formula, the residue theorem, Laurent expansion theorems, etc. carry over to the higher dimensional context using this operator, see for instance $[4,11,12]$. Nevertheless, as far as we know, questions concerning possible generalizations of Wiman-Valiron theory remained untouched for a long time.

In [1] M.A. Abul-Ez and the first author introduced the notion of the growth order and the type for a particular subclass of entire Clifford holomorphic functions. These were called special monogenic functions by the authors and have the property of being generated by a special subfamily of monogenic polynomials. In the follow-up papers $[2,3]$ it was analyzed under which growth conditions the related basic set of special polynomials form a Cannon set. This provided some first results on some questions around the growth of this particular subclass. 
In the recent papers $[5,6,8]$ the fundamentals of a generalized WimanValiron theory for general entire monogenic functions were established. In particular, generalizations of the maximum term and of the central index to Clifford analysis were introduced for general entire monogenic functions. This study lead to more quantitative insight in the asymptotic growth behavior of the maximum modulus and its relation to the Taylor coefficients of general entire monogenic functions.

In this paper we now develop the basics of a higher dimensional generalization of Wiman-Valiron theory for monogenic Taylor series of finite convergence radius.

We first introduce the notions of growth orders in this context and study some basic properties. As described in [8], in the case of entire monogenic functions the set of Fueter polynomials turned out to be the particular set of entire monogenic functions that have growth order equal to zero. In the case of monogenic Taylor series of finite convergence radius, the class of functions with growth order zero is replaced by the set of hypercomplex meromorphic Mittag-Leffler series with isolated singularities discretely placed on the boundary of the unit ball.

After that we study the relation between the growth order of a finite monogenic Taylor series, and the growth order of its partial derivatives.

Then we introduce the maximum term and the central index in this framework and study their relation to generalized growth orders. We set up some generalizations of those estimates between these entities that were discussed in $[19,20]$ for the one variable case. This includes a generalization of the classical Valiron inequality. As the main application we develop explicit formulas for the growth order in terms of the Taylor coefficients of the monogenic function series.

\section{Preliminaries}

In this section we introduce the basic notions of Clifford analysis. For detailed information about Clifford algebras and their function theory we refer for example to [4], [11] and [12].

\subsection{Clifford algebras}

By $\left\{e_{1}, e_{2}, \ldots, e_{n}\right\}$ we denote the canonical basis of the Euclidean vector space $\mathbb{R}^{n}$. The attached real Clifford algebra $\mathbf{C l}_{0 n}$ is the free algebra generated by $\mathbb{R}^{n}$ modulo the relation $\mathbf{x}^{2}=-\|\mathbf{x}\|^{2} e_{0}$, where $\mathbf{x} \in \mathbb{R}^{n}$ and $e_{0}$ is the neutral element with respect to multiplication of the Clifford algebra $\mathbf{C l}_{0 n}$. In the Clifford algebra $\mathbf{C l}_{0 n}$ the following multiplication rules hold $e_{i} e_{j}+e_{j} e_{i}=-2 \delta_{i j} e_{0}$, where $i, j=1, \cdots, n$, and where $\delta_{i j}$ is the Kronecker symbol. A basis for the Clifford algebra $\mathbf{C l}_{0 n}$ is given by the set 
$\left\{e_{A}: A \subseteq\{1, \cdots, n\}\right\}$ with $e_{A}=e_{l_{1}} e_{l_{2}} \cdots e_{l_{r}}$, where $1 \leq l_{1}<\cdots<l_{r} \leq$ $n, \quad e_{\emptyset}=e_{0}=1$. Every $a \in \mathbf{C l}_{0 n}$ can be written in the form $a=\sum_{A} a_{A} e_{A}$ with $a_{A} \in \mathbb{R}$. Two examples of real Clifford algebras are the complex number field $\mathbb{C}$ and the Hamiltonian skew field $\mathbb{H}$. The conjugation antiautomorphism in the Clifford algebra $\mathbf{C l}_{0 n}$ is defined by $\bar{a}=\sum_{A} a_{A} \bar{e}_{A}$, where $\bar{e}_{A}=\bar{e}_{l_{r}} \bar{e}_{l_{r-1}} \cdots \bar{e}_{l_{1}}$ and $\bar{e}_{j}=-e_{j}$ for $j=1, \cdots, n, \bar{e}_{0}=e_{0}=1$. The linear subspace $\operatorname{span}_{\mathbb{R}}\left\{1, e_{1}, \cdots, e_{n}\right\}=\mathbb{R} \oplus \mathbb{R}^{n} \subset \mathbf{C l}_{0 n}$ is the so-called space of paravectors $z=x_{0}+x_{1} e_{1}+x_{2} e_{2}+\cdots+x_{n} e_{n}$ which we simply identify with $\mathbb{R}^{n+1}$. The term $x_{0}$ is called the scalar part of the paravector $z$ and $\mathbf{x}:=x_{1} e_{1}+\cdots+x_{n} e_{n}$ its vector part. Any paravector $z \in \mathbb{R}^{n+1} \backslash\{0\}$ has an inverse element in $\mathbb{R}^{n+1}$ given by $z^{-1}=\bar{z} /\|z\|^{2}$. A scalar product between two Clifford numbers $a, b \in \mathbf{C l}_{0 n}$ is defined by $\langle a, b\rangle:=S c(a \bar{b})$ and the Clifford norm of an arbitrary $a=\sum_{A} a_{A} e_{A}$ is $\|a\|=\left(\sum_{A}\left|a_{A}\right|^{2}\right)^{1 / 2}$.

In order to present many calculations in a more compact form, the following notations will be used, where $\mathbf{m}=\left(m_{1}, \ldots, m_{n}\right) \in \mathbb{N}_{0}^{n}$ is an $n$ dimensional multi index:

$$
\mathbf{x}^{\mathbf{m}}:=x_{1}^{m_{1}} \cdots x_{n}^{m_{n}}, \quad \mathbf{m} !:=m_{1} ! \cdots m_{n} !, \quad|\mathbf{m}|:=m_{1}+\cdots+m_{n} .
$$

By $\tau(i)$ we denote the multi index $\left(m_{1}, \ldots, m_{n}\right)$ with $m_{j}=\delta_{i j}$ for $1 \leq j \leq n$.

\subsection{Clifford analysis}

One way to generalize complex function theory to higher dimensional hypercomplex spaces is the Riemann approach. One considers Clifford algebra valued functions defined in $\mathbb{R}^{n+1}$ that are annihilated by the generalized Cauchy-Riemann operator

$$
D:=\frac{\partial}{\partial x_{0}}+\sum_{i=1}^{n} e_{i} \frac{\partial}{\partial x_{i}} .
$$

If $U \subset \mathbb{R}^{n+1}$ is an open set, then a real differentiable function $f: U \rightarrow \mathbf{C l}_{0 n}$ is called left (right) monogenic at a point $z \in U$ if $D f(z)=0($ or $f D(z)=0)$. Functions that are left monogenic in the whole space are also called left entire monogenic. The notion of left (right) monogenicity in $\mathbb{R}^{n+1}$ actually provides a meaningful generalization of the concept of complex analyticity to Clifford analysis. Many classical theorems from complex analysis could be generalized to higher dimensions by this approach. We refer for example to $[4,11,12]$. One important tool is the generalized Cauchy formula.

Let us denote by $A_{n+1}$ the $n$-dimensional surface 'area' of the $(n+1)$ dimensional unit ball, and by $q_{\mathbf{0}}(z)=\frac{\bar{z}}{\|z\|^{n+1}}$ the Cauchy kernel. Then every function $f$ that is left monogenic in a neighborhood of the closure $\overline{\mathcal{D}}$ of a domain $\mathcal{D}$ satisfies

$$
f(z)=\frac{1}{A_{n+1}} \int_{\partial \mathcal{D}} q_{\mathbf{0}}(z-w) d \sigma(w) f(w),
$$


where $d \sigma(w)$ is the paravector-valued outer normal surface measure, i.e.,

$$
d \sigma(w)=\sum_{j=0}^{n}(-1)^{j+1} e_{j} d w_{0} \wedge \cdots \wedge \widehat{d w_{j}} \wedge \cdots \wedge d w_{n}
$$

with $\widehat{d w_{j}}=d w_{0} \wedge \cdots \wedge d w_{i-1} \wedge d w_{i+1} \wedge \cdots \wedge d w_{n}$

An important difference to classical complex function theory is that the set of left (right) monogenic functions forms only a Clifford right (left) module for $n>1$. The ordinary powers of the hypercomplex variable $z$ are not monogenic, either. In the Clifford analysis setting, the complex positive powers are substituted by Fueter polynomials. These are defined by

$$
\mathcal{P}_{\mathbf{m}}(z)=\frac{1}{|\mathbf{m}| !} \sum_{\pi \in \operatorname{perm}(\mathbf{m})} z_{\pi\left(m_{1}\right)} z_{\pi\left(m_{2}\right)} \cdots z_{\pi\left(m_{n}\right)},
$$

where $\operatorname{perm}(\mathbf{m})$ stands for the set of all permutations of the sequence $\left(m_{1}, m_{2}, \ldots, m_{n}\right)$ and $z_{i}:=x_{i}-x_{0} e_{i}$ for $i=1, \ldots, n$ and $\mathcal{P}_{\mathbf{0}}(z):=1$. For convenience in this paper we prefer to work with the slightly modified Fueter polynomials $V_{\mathbf{m}}(z):=\mathbf{m} ! \mathcal{P}_{\mathbf{m}}(z)$. The negative power functions are generalized by the Cauchy kernel function $q_{\mathbf{0}}(z)$ and its partial derivatives $q_{\mathbf{m}}(z)=\frac{\partial^{m_{1}+\cdots+m_{n}}}{\partial x_{1}^{m_{1}} \cdots \partial x_{n}^{m_{n}}} q_{\mathbf{0}}(z)$. In the generalized Taylor and Laurent expansion theorem, the functions $V_{\mathbf{m}}(z)$ and $q_{\mathbf{m}}(z)$ replace the ordinary powers in complex function theory. To make the paper self-contained we briefly recall

Theorem 1. ([4] pp. 71) Let $g: B(0, R) \rightarrow \mathbf{C l}_{0 n}$ be left monogenic. Then in each open ball $B(0, r)$ with $0<r<R$

$$
g(z)=\sum_{m=0}^{\infty}\left(\sum_{|\mathbf{m}|=m} V_{\mathbf{m}}(z) a_{\mathbf{m}}\right)
$$

where all terms of the same total degree of homogeneity $|\mathbf{m}|$ are bracketed together and the coefficients are given by $a_{\mathbf{m}}=\frac{1}{\mathbf{m} ! A_{n+1}} \int_{\partial B(0, r)} q_{\mathbf{m}}(z) d \sigma(z) g(z)$.

Remarks: Suppose that $f$ is left monogenic in $B(0, R)$ with some singularities on the boundary $\{z:\|z\|=R\}$. Then we know for sure that the Taylor series (1) converges in each $B(0, r)$ with $0 \leq r<R$ uniformely to the function $g$. However, as already mentioned in the old work of Fueter [10] p. 125 , in contrast to the one complex variable case, it can happen that the Taylor series (1) even converges in some points outside the closure of $B(0, R)$. The open ball $B(0, R)$ is only the largest ball centered at the origin that lies completely inside the convergence domain of the series (1). It is important that in the representation (1) the terms of the same total degree of homogeneity are bracketed together. If we consider instead its usual multiple Taylor series representation in which the terms of the same homogeneity degree are not necessarily bracketed together, then it can happen that this one only converges in the smaller domain $\frac{R}{\sqrt{2}}$, see also [9]. As 
a consequence for $n>1$ we do not have the classical Hadamard formula which relates directly the Taylor coefficients with the convergence radius in the one complex variable case. In the Clifford analysis setting one only has an inequality of the form

$$
\frac{1}{R} \leq \limsup _{|\mathbf{m}| \rightarrow+\infty} \sqrt[|\mathbf{m}|]{\left\|a_{\mathbf{m}}\right\|} \leq \frac{\sqrt{2}}{R} .
$$

An example where the lower bound is attained is given by the function $f(z)=\frac{1}{1-x_{0}-e_{1} x_{1}}$. The upper bound limit $\frac{1}{\sqrt{2}}$ is obtained for instance by $g(z)=q_{0}\left(e_{1}-z\right)$.

\section{Growth orders of monogenic functions in the unit ball}

The aim of this paper is to develop analogues of some basic results from Wiman and Valiron's theory for monogenic Taylor series

$$
g(z)=\sum_{m=0}^{\infty}\left(\sum_{|\mathbf{m}|=m} V_{\mathbf{m}}(z) a_{\mathbf{m}}\right)
$$

of finite convergence radius $R$. For the sake of simplicity we will use for (1) in all that follows the abbreviated notation

$$
g(z)=\sum_{|\mathbf{m}|=0}^{\infty} V_{\mathbf{m}}(z) a_{\mathbf{m}}
$$

where we always presuppose that all terms of the same degree are bracketed together. Without loss of generality let us suppose that the convergence radius of (3) is equal to 1 . Throughout this paper we denote the maximum modulus by $M(r, g):=M(r):=\max \{\|g(z)\|:\|z\|=r<1\}$. We define the maximum term of (3) by $\mu(r, g):=\mu(r):=\max \left\{\left\|a_{\mathbf{m}}\right\| r^{|\mathbf{m}|}\right\}$ and the central index (or indices resp.) by $\nu(r, g):=\nu(r):=\max \left\{|\mathbf{m}| ;\left\|a_{\mathbf{m}}\right\| r^{|\mathbf{m}|}:=\right.$ $\mu(r, g)\}$.

Following the classical line from G. Valiron and $[19,20]$, it is natural to define the growth order of a monogenic function in the unit ball as follows:

Definition 1. (Order of growth)

Let $g: B(0,1) \rightarrow \mathbf{C l}_{0 n}$ be a left monogenic function. Then we call

$$
\rho(g):=\limsup _{r \rightarrow 1^{-}} \frac{\log ^{+}\left(\log ^{+} M(r, g)\right)}{-\log (1-r)}, \quad 0 \leq \rho \leq \infty
$$

the order of growth of the function $g$. The inferior growth order of $g$ is defined by

$$
\lambda(g):=\liminf _{r \rightarrow 1^{-}} \frac{\log ^{+}\left(\log ^{+} M(r, g)\right)}{-\log (1-r)}, \quad 0 \leq \lambda \leq \infty .
$$

If $\rho=\lambda$, then we say that $g$ is a function of regular growth. If $\rho>\lambda$ then we say that $g$ has irregular growth. 
A simple example of a non-constant monogenic Taylor series with convergence radius equal to 1 is given by $F(z):=\sum_{|\mathbf{m}|=0}^{+\infty} V_{\mathbf{m}}(z)$. We immediately observe that for $\|z\|=r<1: M(r, F) \leq \sum_{|\mathbf{m}|=0}^{+\infty}\|z\|^{|\mathbf{m}|}=\frac{1}{1-r}$. Hence,

$$
\frac{\log ^{+} \log ^{+} M(r, F)}{-\log (1-r)} \leq \frac{\log ^{+}(-\log (1-r))}{-\log (1-r)} \rightarrow 0 \quad \text { for } r \rightarrow 1^{-} .
$$

We thus have $\rho(F)=0$ and consequently also $\lambda(F)=0$. The function $F$ serves as an example for a monogenic function in the unit ball of regular growth.

Here, we observe a further interesting aspect differing significantly from the case dealing with entire monogenic functions: The subset of entire monogenic functions whose growth order equals zero coincided with the set of monogenic polynomials. Here, in the context of monogenic Taylor series of finite convergence radius, the situation is different. In the setting of the unit ball all finite Mittag-Leffler series with isolated singularities on the unit sphere turn out to have growth order zero.

Theorem 2. Let $a_{1}, \ldots, a_{k}$ be mutually distinct points on the unit sphere $S^{n}=\left\{z \in \mathbb{R}^{n+1} \mid\|z\|=1\right\}, N\left(a_{i}\right)$ some arbitrary non-negative integers and $b_{\mathbf{m}}^{(i)}$ some arbitrary Clifford numbers. Then all Mittag-Leffler series of the form

$$
Q(z)=\sum_{i=1}^{k} \sum_{|\mathbf{m}|=0}^{N\left(a_{i}\right)} q_{\mathbf{m}}\left(a_{i}-z\right) b_{\mathbf{m}}^{(i)}
$$

satisfy $\lambda(Q)=\rho(Q)=0$.

Proof. Let us first consider the particular term $q_{\mathbf{m}}\left(a_{i}-z\right)$. Following e.g. [7], it satisfies the sharp estimate

$$
\left\|q_{\mathbf{m}}\left(a_{i}-z\right)\right\| \leq \frac{n(n+1) \cdots(n+|\mathbf{m}|-1)}{\left\|a_{i}-z\right\|^{n+|\mathbf{m}|}} .
$$

For all $a_{i} \in S^{n}$ and for all $z$ with $\|z\|=r<1$ we have $\left\|a_{i}-z\right\| \geq(1-r)$. Therefore,

$$
M\left(r, q_{\mathbf{m}}\left(a_{i}-z\right)\right) \leq \frac{c_{0}(n,|\mathbf{m}|)}{(1-r)^{n+|\mathbf{m}|}}
$$

with $c_{0}(n,|\mathbf{m}|)=n(n+1) \cdots(n+|\mathbf{m}|-1)$ and hence

$$
\frac{\log ^{+} \log ^{+} M\left(r, q_{\mathbf{m}}\left(a_{i}-z\right)\right)}{-\log (1-r)}=\frac{\log ^{+} \log ^{+}\left(\frac{c_{0}(n,|\mathbf{m}|)}{\left.(1-r)^{n+|\mathbf{m}|}\right)}\right.}{-\log (1-r)} \rightarrow 0 \quad \text { for } r \rightarrow 1^{-} \text {. }
$$

So, we have $\rho\left(q_{\mathbf{m}}\left(a_{i}-z\right)\right)=0$ and $\lambda\left(q_{\mathbf{m}}\left(a_{i}-z\right)\right)=0$. Like in the classical theory of complex holomorphic functions, also two arbitrary monogenic Taylor 
series in the unit ball satisfy $\rho(f+g) \leq \max \{\rho(f), \rho(g)\}$ and $\rho(f \beta)=\rho(f)$ for all arbitrary Clifford numbers $\beta \in \mathbf{C l}_{0 n}$. Hereby follows the assertion.

Next we analyze the relation between the growth behavior of a monogenic Taylor series in the unit ball and the growth order of its partial derivatives. We establish

Theorem 3. Suppose that $g: B(0,1) \rightarrow \mathbf{C l}_{0 n}$ is a left monogenic function. Let further $M_{i}(r):=\max _{\|z\|=r}\left\{\left\|\frac{\partial g}{\partial x_{i}}(z)\right\|\right\}$ where $0<r<1$ and $i \in\{0,1, \ldots, n\}$ and let $M^{\prime}(r):=\max _{i=0,1, \ldots, n} M_{i}(r)$. Then

$$
\rho(g)=\rho^{\prime}(g) \quad \text { and } \quad \lambda(g)=\lambda^{\prime}(g)
$$

where $\rho^{\prime}(g)=\limsup _{r \rightarrow 1^{-}} \frac{\log ^{+} \log ^{+} M^{\prime}(r)}{-\log (1-r)}$ and $\lambda^{\prime}(g)=\liminf _{r \rightarrow 1^{-}} \frac{\log ^{+} \log ^{+} M^{\prime}(r)}{-\log (1-r)}$.

Proof. Since $g$ is left monogenic for $\|z\|<1$, it follows immediately by Cauchy's integral formula for monogenic functions that also all the partial derivatives $g_{i}(z):=\frac{\partial g}{\partial x_{i}}(z)$ for $i=0,1, \ldots, n$ are left monogenic functions for $\|z\|<1$. Let $\Gamma^{\prime}:[0,1] \rightarrow B(0,1)$ be an arbitrary rectifiable curve within the unit ball starting from the origin to $z$. Then, one can write $g(z)=g(0)+\int_{0}^{1} \sum_{i=0}^{n} x_{i} g_{i}(t z) d t$. For all $\|z\|=r<1$ we obtain

$$
\|g(z)\| \leq\|g(0)\|+r(n+1) M^{\prime}(r)
$$

from which one can directly conclude that $\rho(g) \leq \rho^{\prime}(g)$ and $\lambda(g) \leq \lambda^{\prime}(g)$.

To prove the estimate in the opposite direction we first apply as in the case of entire monogenic functions Cauchy's integral formula on the partial derivatives $g_{i}$. For all $0<r<R<1$ we obtain

$$
g_{i}(z)=\frac{1}{A_{n+1}} \int_{\|\zeta-z\|=R-r} q_{\tau(i)}(\zeta-z) d \sigma(\zeta) g(\zeta) .
$$

In view of $\left\|q_{\tau(i)}(\zeta-z)\right\| \leq \frac{n}{(R-r)^{n+1}}$ we hence get for all $0<r<R<1$ the estimate

$$
M^{\prime}(r) \leq \frac{n}{R-r} M(R) .
$$

which is the same as in the case of entire monogenic functions. However, in order to proceed further, we now need to apply different arguments as in the case of entire functions.

It is convenient to set: $R=\alpha r$ where $\alpha \in \mathbb{R}$ has to be chosen such that $1<\alpha<\frac{1}{r}$ where $r<1$. Then (8) reads $M^{\prime}(r) \leq \frac{n}{(\alpha-1) r} M(\alpha r)$, so that

$$
\log ^{+} M^{\prime}(r) \leq \log ^{+} M(\alpha r)+\log ^{+} \frac{n}{(\alpha-1) r} .
$$


Now we have

$$
\begin{aligned}
\frac{\log ^{+} \log ^{+} M^{\prime}(r)}{-\log (1-r)} & \leq \frac{\log ^{+}\left[\log ^{+} M(\alpha r)+\log ^{+}\left(\frac{n}{(\alpha-1) r}\right)\right]}{-\log (1-\alpha r)} \frac{\log (1-\alpha r)}{\log (1-r)} \\
& \leq \frac{\log ^{+} \log ^{+} M(\alpha r)}{-\log (1-\alpha r)} \frac{\log (1-\alpha r)}{\log (1-r)} \\
& +\frac{\log ^{+} \log ^{+}\left(\frac{n}{(\alpha-1) r}\right)}{-\log (1-\alpha r)} \frac{\log (1-\alpha r)}{\log (1-r)}+\frac{\log 2}{\log (1-r)} .
\end{aligned}
$$

Let us now investigate the asymptotic behavior of these terms for $r \rightarrow 1^{-}$. If $r$ tends to $1^{-}$, then it follows from $1<\alpha<\frac{1}{r}$ that $\alpha \approx 1$. Therefore, the expression $\frac{\log ^{+} \log ^{+}\left(\frac{n}{(\alpha-1) r}\right)}{-\log (1-\alpha r)}$ has the same asymptotic behavior as $\frac{\log \log n x}{\log x}$ for

very large $x$. This in turn tends to zero. Furthermore, $\frac{\log (1-\alpha r)}{\log (1-r)} \approx 1$. As a consequence we obtain that $\rho(g) \geq \rho^{\prime}(g)$ and $\lambda(g) \geq \lambda^{\prime}(g)$.

\section{Relations between the growth order and the Taylor coefficients}

In analogy to the classical Valiron inequality [13,21] for holomorphic functions we obtain the following generalization in the setting of monogenic Taylor series of finite convergence radius:

Theorem 4. If $g: \mathbb{R}^{n+1} \rightarrow \mathbf{C l}_{0 n}$ is a left monogenic function in $B(0,1)$, then we have for all $0<r<R<1$ :

$$
M(r) \leq \mu(r)\left[\left(\begin{array}{c}
n+|\nu(R)|-1 \\
|\nu(R)|-1
\end{array}\right)+\left(\frac{1}{R-r}\right)^{n}\right] .
$$

Proof. Since $g$ is monogenic in the open ball $B(0,1)$, it has the representation $g(z)=\sum_{|\mathbf{1}|=0}^{\infty} V_{\mathbf{l}}(z) a_{\mathbf{l}}$ for all $\|z\|<1$ where $a_{\mathbf{l}}$ are uniquely defined Clifford numbers. From the maximum modulus theorem for monogenic functions we infer that for $0<r<R<1$ :

$$
\begin{aligned}
M(r) & \leq \sum_{|\mathbf{1}|=0}^{\infty}\left\|a_{\mathbf{l}}\right\| r^{|\mathbf{1}|}=\sum_{|\mathbf{1}|=0}^{|\nu(R)|-1}\left\|a_{\mathbf{l}}\right\| r^{|\mathbf{1}|}+\sum_{|\mathbf{1}|=|\nu(R)|}^{\infty}\left\|a_{\mathbf{l}}\right\| r^{|\mathbf{1}|} \\
& \leq \sum_{|\mathbf{1}|=0}^{|\nu(R)|-1} \mu(r)+\sum_{|\mathbf{1}|=|\nu(R)|}^{\infty}\left\|a_{\mathbf{l}}\right\| r^{|\mathbf{1}|} .
\end{aligned}
$$

In view of $\sum_{|\mathbf{1}|=0}^{|\nu(R)|-1} 1=\left(\begin{array}{c}n+|\nu(R)|-1 \\ |\nu(R)|-1\end{array}\right)$ we obtain after inserting this expression into (10):

$$
M(r) \leq \mu(r)\left(\begin{array}{c}
n+|\nu(R)|-1 \\
|\nu(R)|-1
\end{array}\right)+\sum_{|\mathbf{l}|=|\nu(R)|}^{\infty}\left\|a_{\mathbf{l}}\right\| r^{|\mathbf{1}|} \frac{\left\|a_{\nu(r)}\right\| r^{|\nu(r)|} R^{|\mathbf{l}+\nu(R)|}}{\left\|a_{\nu(r)}\right\| r^{|\nu(R)|} R^{|\mathbf{l}+\nu(R)|}}
$$




$$
\begin{aligned}
& \leq \mu(r)\left(\begin{array}{c}
n+|\nu(R)|-1 \\
|\nu(R)|-1
\end{array}\right)+\mu(r) \sum_{|\mathbf{1}|=|\nu(R)|}^{\infty} \frac{\left.\left\|a_{\mathbf{l}}\right\| R^{|\mathbf{1}|} R^{|\nu(R)|}\right|^{|\mathbf{1}|}}{\left\|a_{\nu(R)}\right\| R^{|\nu(R)|} R^{|\mathbf{1}|} r^{|\nu(R)|}} \\
& \leq \mu(r)\left(\begin{array}{c}
n+|\nu(R)|-1 \\
|\nu(R)|-1
\end{array}\right)+\mu(r) \sum_{|\mathbf{1}|=|\nu(R)|}^{\infty}\left(\frac{r}{R}\right)^{|\mathbf{1}|-|\nu(R)|} \\
& =\mu(r)\left[\left(\begin{array}{c}
n+|\nu(R)|-1 \\
|\nu(R)|-1
\end{array}\right)+\left(\frac{R}{R-r}\right)^{n}\right] \\
& \leq \mu(r)\left[\left(\begin{array}{c}
n+|\nu(R)|-1 \\
|\nu(R)|-1
\end{array}\right)+\left(\frac{1}{R-r}\right)^{n}\right] .
\end{aligned}
$$

For the applications that we are going to develop in this paper it suffices to use the following weaker version which also provides a correction to Theorem 5.2 from [8]:

Corollary 1. If $g: \mathbb{R}^{n+1} \rightarrow \mathbf{C l}_{0 n}$ is a left monogenic function in $B(0,1)$, then for all $0<r<R<1$ :

$$
M(r) \leq \mu(r)\left[|\nu(R)|(1+|\nu(R)|)^{n-1}+\left(\frac{1}{R-r}\right)^{n}\right] .
$$

Proof. It suffices to show that

$$
\left(\begin{array}{c}
n+|\nu(R)|-1 \\
|\nu(R)|-1
\end{array}\right) \leq|\nu(R)|\left[(1+|\nu(R)|)^{n-1}\right] .
$$

In view of $\left(\begin{array}{c}n+|\nu(R)|-1 \\ |\nu(R)|-1\end{array}\right)=\sum_{|\mathbf{1}|=0}^{|\nu(R)|-1} 1$, we obtain the following estimate

$$
\begin{aligned}
\sum_{|\mathbf{1}|=0}^{|\nu(R)|-1} 1 & =\sum_{|\mathbf{1}|=0} 1+\sum_{|\mathbf{1}|=1} 1+\cdots+\sum_{|\mathbf{1}|=|\nu(R)|-1} 1 \\
& =1+\frac{((n-1)+1) !}{(n-1) ! 1 !}+\cdots+\frac{[(n-1)+(\mid \nu(R)-1)] !}{(n-1) !(|\nu(R)|-1) !} \\
& \leq|\nu(R)|\left[\frac{[(n-1)+|\nu(R)|-1] !}{(n-1) !(|\nu(R)|-1) !}\right]
\end{aligned}
$$

where we used the inequality

$$
\frac{(n-1+k) !}{(n-1) ! k !} \leq \frac{(n-1+(k+1)) !}{(n-1) !(k+1) !}
$$

which in turn can be established by a straightforward induction argument for all positive integers $n \geq 1$. Further,

$$
|\nu(R)|\left[\frac{[(n-1)+|\nu(R)|-1] !}{(n-1) !(|\nu(R)|-1) !}\right]
$$




$$
\begin{aligned}
& =|\nu(R)|\left[\frac{(|\mu(R)|+n-2)((|\nu(R)|+n-3) \cdots(|\nu(R)|+1)|\nu(R)|}{(n-1) !}\right] \\
& =|\nu(R)|\left[\frac{|\nu(R)|+n-2}{n-1} \cdot \frac{|\nu(R)|+n-3}{n-2} \cdots \cdots \frac{|\nu(R)|+1}{2} \frac{|\nu(R)|}{1}\right] \\
& \leq|\nu(R)|[\underbrace{\left(1+\frac{|\nu(R)|}{n-1}\right)}_{\leq 1+|\nu(R)|} \underbrace{\left.1+\frac{|\nu(R)|}{n-2}\right) \cdots(\underbrace{\left.1+\frac{|\nu(R)|}{1}\right)}_{=1+|\nu(R)|}]}_{\leq 1+|\nu(R)|} \\
& \leq|\nu(R)|\left[(1+|\nu(R)|)^{n-1}\right] .
\end{aligned}
$$

Finally, one obtains:

$$
M(r) \leq \mu(r)\left[|\nu(R)|(1+|\nu(R)|)^{n-1}+\frac{1}{(R-r)^{n}}\right] .
$$

As a direct consequence we further obtain

Corollary 2. If $g: \mathbb{R}^{n+1} \rightarrow \mathbf{C l}_{0 n}$ is a non-constant left monogenic function in $B(0,1)$, then for all $0<r<R<1$ and $R:=r+\frac{1-r}{|\nu(r)|}$

$$
M(r)<2 \mu(r)(1+|\nu(R)|)^{n} \frac{1}{(1-r)^{n}} .
$$

Proof. Inserting the particular value $R:=r+\frac{1-r}{|\nu(r)|}$ into inequality (11) leads to

$$
M(r) \leq \mu(r)\left[|\nu(R)|(1+|\nu(R)|)^{n-1}+\frac{|\nu(r)|^{n}}{(1-r)^{n}}\right] .
$$

Since $|\nu|$ is an increasing function, we get

$$
\begin{aligned}
M(r) & \leq \mu(r)\left((1+|\nu(R)|)^{n}+\frac{(1+|\nu(R)|)}{(1-r)^{n}}\right) \\
& =\mu(r)(1+|\nu(R)|)^{n}\left(1+\frac{1}{(1-r)^{n}}\right)<2 \mu(r)(1+|\nu(R)|)^{n} \frac{1}{(1-r)^{n}} .
\end{aligned}
$$

Our next aim is to establish some relations between the order of growth $\rho$ and the expressions

$$
\begin{array}{ll}
\rho_{1}:=\limsup _{r \rightarrow 1^{-}} \frac{\log ^{+} \log ^{+} \mu(r)}{-\log (1-r)} ; & \rho_{2}:=\limsup _{r \rightarrow 1^{-}} \frac{\log ^{+}|\nu(r)|}{-\log (1-r)} ; \\
\lambda_{1}:=\liminf _{r \rightarrow 1^{-}} \frac{\log ^{+} \log ^{+} \mu(r)}{-\log (1-r)} ; & \lambda_{2}:=\liminf _{r \rightarrow 1^{-}} \frac{\log ^{+}|\nu(r)|}{-\log (1-r)} .
\end{array}
$$

The generalized Valiron inequality allows us to establish 
Theorem 5. Let $g: \mathbb{R}^{n+1} \rightarrow \mathbf{C l}_{0 n}$ be a left monogenic function in $B(0,1)$ of growth order $\rho$. If $\rho_{2}<\infty$ then $\rho \leq \rho_{1}$.

Proof. Inserting the particular value $R=\alpha r$ with $1<\alpha<\frac{1}{r}$ into (11) leads to

$$
M(r) \leq \mu(r)\left[|\nu(\alpha r)|[1+|\nu(\alpha r)|]^{n-1}+\frac{1}{((\alpha-1) r)^{n}}\right] .
$$

In view of $\rho_{2}<\infty$ we can always find an $\varepsilon>0$ such that $\frac{\log |\nu(r)|}{-\log (1-r)} \leq \rho_{2}+\varepsilon$, which equivalently reads $|\nu(r)| \leq e^{-\left(\rho_{2}+\varepsilon\right) \log (1-r)}=(1-r)^{-\left(\rho_{2}+\varepsilon\right)}$. For $r$ sufficiently close to $1^{-}$there is an $\varepsilon_{1}>0$ such that

$$
M(r) \leq \mu(r)\left((1-\alpha r)^{-n\left(\rho_{2}+\varepsilon\right)}+\frac{1}{((\alpha-1) r)^{n}}\right) \leq \mu(r)(1-\alpha r)^{-n\left(\rho_{2}+\varepsilon_{1}\right)} .
$$

Hence with $\varepsilon_{2}:=n \varepsilon_{1}$ we obtain $M(r) \leq \mu(r)(1-\alpha r)^{-n \rho_{2}-\varepsilon_{2}}$. Next we may infer that

$$
\begin{aligned}
\frac{\log ^{+} \log ^{+} M(r)}{-\log (1-r)} & \leq \frac{\log ^{+}\left[\log ^{+} \mu(r)+\log ^{+}(1-\alpha r)^{-n \rho_{2}-\varepsilon_{2}}\right]}{-\log (1-r)} \\
& =\frac{\log 2}{-\log (1-r)}+\frac{\log ^{+}\left(\left(-n \rho_{2}-\varepsilon_{2}\right) \log (1-\alpha r)\right)}{-\log (1-r)} \\
& +\frac{\log ^{+} \log ^{+} \mu(r)}{-\log (1-r)} .
\end{aligned}
$$

For $r \rightarrow 1^{-}$we have $\alpha \approx 1$. Hence $\lim _{r \rightarrow 1^{-}} \frac{\log ^{+}\left(-\left(n \rho_{2}+\varepsilon_{2}\right) \log (1-\alpha r)\right)}{-\log (1-r)}=0$ and therefore

$$
\rho=\limsup _{r \rightarrow 1^{-}} \frac{\log ^{+} \log ^{+} M(r)}{-\log (1-r)} \leq \limsup _{r \rightarrow 1^{-}} \frac{\log ^{+} \log ^{+} \mu(r)}{-\log (1-r)}=\rho_{1} .
$$

Proposition 1. Suppose that $g: B(0,1) \rightarrow \mathbf{C l}_{0 n}$ is a left monogenic function represented by $g(z)=\sum_{|\mathbf{m}|=0}^{\infty} V_{\mathbf{m}}(z) a_{\mathbf{m}}$. Further let

$$
\kappa:=\limsup _{|\mathbf{m}| \rightarrow \infty} \frac{\log ^{+} \log ^{+}\left(\left\|a_{\mathbf{m}}\right\|\right)}{\log (|\mathbf{m}|)} \text { and } \omega:=\liminf _{|\mathbf{m}| \rightarrow \infty} \frac{\log ^{+} \log ^{+}\left(\left\|a_{\mathbf{m}}\right\|\right)}{\log (|\mathbf{m}|)}
$$

Then $\rho_{1} \leq \kappa \rho_{2}$ and $\lambda_{1} \leq \omega \lambda_{2}$.

Proof. We consider $\mu(r):=\left\|a_{\nu(r)}\right\| r^{|\nu(r)|}$ for $0<r<1$. Relying on

$$
\log ^{+}(\mu(r))=\log ^{+}\left\|a_{\nu(r)}\right\|+|\nu(r)| \log ^{+}(r)=\log ^{+}\left\|a_{\nu(r)}\right\|
$$

and on the fact that $\kappa$ is finite it follows that

$$
\frac{\log ^{+} \log ^{+}\left(\left\|a_{\mathbf{m}}\right\|\right)}{\log (|\mathbf{m}|)} \leq \kappa+\xi
$$


for $\xi>0$. The inequality (16) equivalently reads

$$
\log ^{+}\left\|a_{\mathbf{m}}\right\| \leq|\mathbf{m}|^{\kappa+\xi}
$$

If we next insert (17) into (15), then we obtain $\log ^{+}(\mu(r)) \leq|\nu(r)|^{\kappa+\varepsilon}$. Applying once more $\log ^{+}$, we get:

$$
\frac{\log ^{+} \log ^{+}(\mu(r))}{-\log (1-r)} \leq \frac{(\kappa+\varepsilon) \log ^{+}(|\nu(r)|)}{-\log (1-r)} .
$$

Consequently, $\lim \sup _{r \rightarrow 1^{-}} \frac{\log ^{+} \log ^{+}(\mu(r))}{-\log (1-r)} \leq \kappa \lim \sup _{r \rightarrow 1^{-}} \frac{\log ^{+}(|\nu(r)|)}{-\log (1-r)}$.

Analogously one can establish that $\lambda_{1} \leq \omega \lambda_{2}$.

Next we establish an explicit relation between the growth order $\rho$ and the Taylor coefficients. The following two theorems provide us with a generalization of the formulas for the complex one variable case proved in [16].

Theorem 6. Suppose that $g: B(0,1) \rightarrow \mathbf{C l}_{0 n}$ is a left monogenic function represented by

$$
g(z)=\sum_{|\mathbf{m}|=0}^{\infty} V_{\mathbf{m}}(z) a_{\mathbf{m}}, \quad\|z\|<1 .
$$

Then the growth order $\rho$ of $g$ is given by

$$
\limsup _{|\mathbf{m}| \rightarrow \infty} \frac{\log ^{+} \log ^{+}\left(\frac{\left\|a_{\mathbf{m}}\right\|}{c(n,|\mathbf{m}|)}\right)}{\log |\mathbf{m}|}=\frac{\rho}{\rho+1}
$$

where $c(n,|\mathbf{m}|):=\frac{n(n+1) \cdots(n+|\mathbf{m}|-1)}{\mathbf{m} !}$.

Proof. In view of the definition of $\rho$ we have $M(r, g)=O\left(e^{(1-r)^{-\alpha}}\right)$ for some $\alpha \in \mathbb{R}$ when $r \rightarrow 1^{-}$and $\rho$ is the infimum of the set of all admissible real $\alpha$. From the integral representation of the Taylor coefficients given in Theorem 1, which is a direct consequence of Cauchy's integral formula, one obtains the following estimate:

$$
\left\|a_{\mathbf{m}}\right\| r^{|\mathbf{m}|} \leq c(n,|\mathbf{m}|) M(r, g) .
$$

Therefore, applying the estimate $\log ^{+} M(r) \leq k(1-r)^{-\alpha}$ where $\alpha>0$ and where $k$ is a properly chosen real constant, leads to

$$
\log ^{+}\left(\frac{\left\|a_{\mathbf{m}}\right\|}{c(n,|\mathbf{m}|)}\right) \leq \log ^{+} M(r, g)+\log r^{-|\mathbf{m}|} \leq k(1-r)^{-\alpha}-|\mathbf{m}| \log r .
$$

For $|\mathbf{m}|>k$ we put

$$
1-r:=\left(\frac{k}{|\mathbf{m}|}\right)^{\frac{1}{1+\alpha}}
$$


Inserting (23) into (22) leads to

$$
\begin{aligned}
\log ^{+}\left(\frac{\left\|a_{\mathbf{m}}\right\|}{c(n,|\mathbf{m}|)}\right) & \leq k\left(\frac{k}{|\mathbf{m}|}\right)^{-\frac{\alpha}{\alpha+1}}-|\mathbf{m}| \log \left(1-\left(\frac{k}{|\mathbf{m}|}\right)^{\frac{1}{1+\alpha}}\right) \\
& \leq 2 k^{\frac{1}{\alpha+1}}|\mathbf{m}|^{\frac{\alpha}{\alpha+1}}-|\mathbf{m}| \log \left(1-\left(\frac{k}{|\mathbf{m}|}\right)^{\frac{1}{1+\alpha}}\right) \\
& =k^{\frac{1}{\alpha+1}}|\mathbf{m}|^{\frac{\alpha}{\alpha+1}} \\
& \times\left(1+1-k^{\frac{-1}{\alpha+1}}|\mathbf{m}|^{\frac{-\alpha}{\alpha+1}}|\mathbf{m}| \log \left(1-\left(\frac{k}{|\mathbf{m}|}\right)^{\frac{1}{1+\alpha}}\right)\right) \\
& =k^{\frac{1}{\alpha+1}}|\mathbf{m}|^{\frac{\alpha}{\alpha+1}}\left(1+O\left(|\mathbf{m}|^{\frac{-1}{1+\alpha}}\right)\right)
\end{aligned}
$$

Moreover,

$$
\limsup _{|\mathbf{m}| \rightarrow \infty} \frac{\log ^{+} \log ^{+}\left(\frac{\left\|a_{\mathbf{m}}\right\|}{c(n,|\mathbf{m}|)}\right)}{\log |\mathbf{m}|} \leq \frac{\alpha}{1+\alpha} .
$$

If $g$ is of finite growth order $\rho$, then one can take any $\alpha \geq \rho$ so that

$$
\limsup _{|\mathbf{m}| \rightarrow \infty} \frac{\log ^{+} \log ^{+}\left(\frac{\left\|a_{\mathbf{m}}\right\|}{c(n,|\mathbf{m}|)}\right)}{\log |\mathbf{m}|} \leq \frac{\rho}{1+\rho} .
$$

Suppose now that $\rho=\infty$.

From the inequalities $(2)$ and in view of $c(n,|\mathbf{m}|) \geq 1$ it follows that $\log \left(\frac{\left|a_{\mathbf{m}}\right|}{c(n,|\mathbf{m}|)}\right) \leq \log \left(\left|a_{\mathbf{m}}\right|\right) \leq|\mathbf{m}| \log \sqrt{2}$.

Thus, $\limsup _{|\mathbf{m}| \rightarrow+\infty} \frac{\log ^{+} \log ^{+}\left(\frac{\left\|a_{\mathbf{m}}\right\|}{c(n,|\mathbf{m}|)}\right)}{\log |\mathbf{m}|} \leq 1$.

If $\rho \neq \infty$, then there are constants $\omega$ and $\beta:=\frac{\omega}{\omega+1}$, such that

$$
\log ^{+}\left\|a_{\mathbf{m}}\right\| \leq|\mathbf{m}|^{\beta}
$$

where $0<\beta<1$. Now we consider those indices with $|\mathbf{m}| \geq\left|\mathbf{m}_{0}\right|$. From (27) we may infer that $\left\|a_{\mathbf{m}}\right\| \leq e^{|\mathbf{m}|^{\beta}}$. Therefore, taking the maximum modulus over $\|z\|=r \leq 1$ in (19), we obtain

$$
M(r) \leq A+\sum_{|\mathbf{m}|=\left|\mathbf{m}_{0}\right|}^{|\mathbf{n}|} e^{|\mathbf{m}|^{\beta}} r^{|\mathbf{m}|}+\sum_{|\mathbf{m}|=|\mathbf{n}|+1}^{\infty} e^{|\mathbf{m}|^{\beta}} r^{|\mathbf{m}|} .
$$

Let us consider those indices $\mathbf{m}$ which satisfy $|\mathbf{n}| \leq|\mathbf{m}| \leq\left|\mathbf{m}_{0}\right|$. We have

$$
\log \left(e^{|\mathbf{m}|^{\beta}} r^{|\mathbf{m}|}\right)=|\mathbf{m}|^{\beta}+|\mathbf{m}| \log r .
$$


Furthermore, in view of

$$
\max _{0 \leq x<\infty}\left(x^{\beta}-x \log \frac{1}{r}\right)=\left(\beta^{\frac{\beta}{1-\beta}}-\beta^{\frac{1}{1-\beta}}\right)\left(\log \left(\frac{1}{r}\right)\right)^{-\omega},
$$

one gets

$$
\log \left(e^{|\mathbf{m}|^{\beta}} r^{|\mathbf{m}|}\right)=\phi(\beta)\left(\log \left(\frac{1}{r}\right)\right)^{-\omega}
$$

where we put $\phi(\beta):=\left(\beta^{\frac{\beta}{1-\beta}}-\beta^{\frac{1}{1-\beta}}\right)$. Inserting (29) into (28) leads to

$$
M(r) \leq A+|\mathbf{n}| e^{\phi(\beta)\left(\log \left(\frac{1}{r}\right)\right)^{-\omega}}+\sum_{|\mathbf{m}|=|\mathbf{n}|+1}^{\infty} e^{|\mathbf{m}|^{\beta}} r^{|\mathbf{m}|}
$$

Consider now $\sum_{|\mathbf{m}|=|\mathbf{n}|+1}^{\infty} e^{|\mathbf{m}|^{\beta}} r^{|\mathbf{m}|}$. Let us consider the greatest integer that does not exceed the value $\left(\frac{1}{2} \log \left(\frac{1}{r}\right)\right)^{\frac{-1}{1-\beta}}$. It will be denoted by

$$
|\mathbf{n}|:=\left[\left(\frac{1}{2} \log \left(\frac{1}{r}\right)\right)^{\frac{-1}{1-\beta}}\right]
$$

where [.] stands for the floor function here. Since $|\mathbf{m}| \geq|\mathbf{n}|+1$ we have

$$
|\mathbf{m}| \geq\left(\frac{2}{-\log (r)}\right)^{\frac{1}{1-\beta}}
$$

(32) is equivalent to $|\mathbf{m}|^{1-\beta} \geq \frac{2}{-\log (r)}$ and consequently to

$$
|\mathbf{m}|^{\beta}-|\mathbf{m}| \log (r) \leq-\frac{|\mathbf{m}|}{2} \log (r)
$$

since

$$
|\mathbf{m}|\left(|\mathbf{m}|^{\beta-1}-\frac{1}{2} \log (r)\right) \leq 0 .
$$

Applying (33) to (30), we obtain

$$
\begin{aligned}
\sum_{|\mathbf{m}|=|\mathbf{n}|+1}^{\infty} e^{|\mathbf{m}|^{\beta}} r^{|\mathbf{m}|} & \leq \sum_{|\mathbf{m}|=|\mathbf{n}|+1}^{\infty} e^{-\frac{|\mathbf{m}|}{2} \log (r)} r^{|\mathbf{m}|} \\
& =\sum_{|\mathbf{m}|=|\mathbf{n}|+1}^{\infty} r^{\frac{|\mathbf{m}|}{2}} \leq \frac{r^{\frac{|\mathbf{n}|+1}{2}}}{1-r^{\frac{1}{2}}} .
\end{aligned}
$$


Put $\omega:=\frac{\beta}{1-\beta}$. In view of $1+|\mathbf{n}| \geq\left(\frac{2}{-\log (r)}\right)^{\frac{1}{1-\beta}}$ we obtain

$$
\begin{aligned}
\log \left(r^{\frac{|\mathbf{n}|+1}{2}}\right) & \leq-\frac{1}{2}\left(\frac{2}{-\log (r)}\right)^{\frac{1}{1-\beta}} \log \frac{1}{r} \\
& \leq-2^{\frac{1}{1-\beta}-1}\left(\log \left(\frac{1}{r}\right)\right)^{1-\frac{1}{1-\beta}} \\
& =-2^{\omega}\left(\log \left(\frac{1}{r}\right)\right)^{-\omega}
\end{aligned}
$$

When $r$ tends to $1^{-}$we have

$$
1-\sqrt{r}=\frac{1}{2} \log \left(\frac{1}{r}\right)\left[1+O\left(\log \left(\frac{1}{r}\right)\right)\right],
$$

and thus obtain

$$
\begin{aligned}
\log \left(\frac{r^{\frac{|\mathbf{n}|+\mathbf{1}}{2}}}{1-\sqrt{r}}\right) & \leq-2^{\omega}\left(\log \left(\frac{1}{r}\right)\right)^{-\omega}+\log 2-\log \log \frac{1}{r}+O\left(\log \frac{1}{r}\right) \\
& =-2^{\omega}\left(\log \left(\frac{1}{r}\right)\right)^{-\omega}[1+o(1)] .
\end{aligned}
$$

Consequently from (36) it follows that (35) is of order $o(1)$ when $r$ tends to $1^{-}$. Finally, inserting (31) into (30) leads to

$$
M(r) \leq A_{1}+\left(\frac{2}{\log \frac{1}{r}}\right)^{\frac{1}{1-\beta}} e^{\phi(\beta)\left(\log \frac{1}{r}\right)^{-\omega}},
$$

where $A_{1}$ is a suitable real constant. Moreover, from the representation $1-r=\left(\log \frac{1}{r}\right)^{-\omega}[1+o(1)]$ we obtain

$$
\begin{aligned}
\log M(r) & \leq \log A_{1}+\frac{1}{1-\beta} \log \left(\frac{2}{\log \frac{1}{r}}\right)+\phi(\beta)\left(\log \frac{1}{r}\right)^{-\omega} \\
& \leq \phi(\beta)\left(\log \frac{1}{r}\right)^{-\omega}[1+o(1)] \\
& \leq \phi(\beta)(1-r)^{-\omega}[1+o(1)] .
\end{aligned}
$$

Furthermore,

$$
\log \log M(r) \leq \log \phi(\beta)+\log \left((1-r)^{-\omega}\right)+O(1) \leq \omega \log \frac{1}{1-r}+O(1),
$$

which leads to

$$
\rho=\lim _{r \rightarrow 1^{-}} \frac{\log \log M(r, f)}{\log \frac{1}{1-r}} \leq \omega \lim _{r \rightarrow 1^{-}} \frac{\log \frac{1}{1-r}}{\log \frac{1}{1-r}}=\omega .
$$


In view of $(27)$ and since $c(n,|\mathbf{m}|) \geq 1$ one has

$$
\log ^{+}\left(\frac{\left\|a_{\mathbf{m}}\right\|}{c(n,|\mathbf{m}|)}\right) \leq \log ^{+}\left\|a_{\mathbf{m}}\right\| \leq|\mathbf{m}|^{\frac{\omega}{1+\omega}}
$$

and therefore

$$
\limsup _{|\mathbf{m}| \rightarrow \infty} \frac{\log \log ^{+}\left(\frac{\left\|a_{\mathbf{m}}\right\|}{c(n,|\mathbf{m}|)}\right)}{\log (|\mathbf{m}|)} \leq \frac{\omega}{1+\omega}
$$

Next we choose the value $\frac{\omega}{1+\omega}$ such that we have for $\varepsilon>0$

$$
\chi:=\limsup _{|\mathbf{m}| \rightarrow \infty} \frac{\log \log ^{+}\left(\frac{\left\|a_{\mathbf{m}}\right\|}{c(n,|\mathbf{m}|)}\right)}{\log (|\mathbf{m}|)}=\frac{\omega}{1+\omega}-\varepsilon .
$$

Since $\rho \leq \omega$ it follows that $\frac{\chi+\varepsilon}{1+(\chi+\varepsilon)}=\omega \geq \rho$ and therefore $\frac{\rho}{\rho+1} \leq \chi+\varepsilon$, i.e.,

$$
\frac{\rho}{1+\rho} \leq \limsup _{|\mathbf{m}| \rightarrow \infty} \frac{\log \log ^{+}\left(\frac{\left\|a_{\mathbf{m}}\right\|}{c(n,|\mathbf{m}|)}\right)}{\log (|\mathbf{m}|)}
$$

From (26) and (37) we finally obtain the formula (20).

This theorem allows us to establish the following explicit formula for the growth order $\rho$ :

Theorem 7. Suppose that $g: B(0,1) \rightarrow \mathbf{C l}_{0 n}$ is a left monogenic function represented in the form (19). Then the growth order $\rho$ of $g$ is given by

$$
\limsup _{|\mathbf{m}| \rightarrow \infty} \frac{\log ^{+} \log ^{+}\left(\frac{\left\|a_{\mathbf{m}}\right\|}{c(n,|\mathbf{m}|)}\right)}{\log |\mathbf{m}|-\log ^{+} \log ^{+}\left(\frac{\left\|a_{\mathbf{m}}\right\|}{c(n,|\mathbf{m}|)}\right)}=\rho .
$$

Proof. Using Theorem 6 one has

$$
\limsup _{|\mathbf{m}| \rightarrow \infty} \frac{\log ^{+} \log ^{+}\left(\frac{\left\|a_{\mathbf{m}}\right\|}{c(n,|\mathbf{m}|)}\right)}{\log |\mathbf{m}|}=\frac{\rho}{\rho+1}
$$

In view of

$$
\frac{\log ^{+} \log ^{+}\left(\frac{\left\|a_{\mathbf{m}}\right\|}{c(n,|\mathbf{m}|)}\right)}{\log |\mathbf{m}|-\log ^{+} \log ^{+}\left(\frac{\left\|a_{\mathbf{m}}\right\|}{c(n,|\mathbf{m}|)}\right)} \approx \frac{1}{\frac{\log (\mid \mathbf{m}) \mid}{\log ^{+} \log ^{+}\left(\frac{\left\|a_{\mathbf{m}}\right\|}{c(n,|\mathbf{m}|)}\right)}-1}
$$

one obtains

$$
\limsup _{|\mathbf{m}| \rightarrow \infty} \frac{\log ^{+} \log ^{+}\left(\frac{\left\|a_{\mathbf{m}}\right\|}{c(n,|\mathbf{m}|)}\right)}{\log |\mathbf{m}|-\log ^{+} \log ^{+}\left(\frac{\left\|a_{\mathbf{m}}\right\|}{c(n,|\mathbf{m}|)}\right)}=\rho
$$

As a direct consequence we can establish 
Corollary 3. Suppose that $g: B(0,1) \rightarrow \mathbf{C l}_{0 n}$ is a left monogenic function represented in the form (19) and let $\rho$ denote the growth order of $g$. Then

$$
\limsup _{|\mathbf{m}| \rightarrow \infty} \frac{\log ^{+} \log ^{+}\left(\left\|a_{\mathbf{m}}\right\|\right)}{\log |\mathbf{m}|} \geq \frac{\rho}{\rho+1} .
$$

Proof. Applying Theorem 6 and $\log \left(\frac{\left|a_{\mathbf{m}}\right|}{c(n,|\mathbf{m}|)}\right) \leq \log \left(\left|a_{\mathbf{m}}\right|\right)$ leads to

$$
\frac{\rho}{\rho+1}=\limsup _{|\mathbf{m}| \rightarrow \infty} \frac{\log ^{+} \log ^{+}\left(\frac{\left|a_{\mathbf{m}}\right|}{c(n,|\mathbf{m}|)}\right)}{\log |\mathbf{m}|} \leq \limsup _{|\mathbf{m}| \rightarrow \infty} \frac{\log ^{+} \log ^{+}\left(\left\|a_{\mathbf{m}}\right\|\right)}{\log |\mathbf{m}|} .
$$

Finally, applying Theorem 6 one also obtains an explicit relation between the growth order $\rho$ and the other entities $\rho_{1}$ and $\rho_{2}$ :

Theorem 8. Suppose that $g: B(0,1) \rightarrow \mathbf{C l}_{0 n}$ is a left monogenic function, where $\rho$ is the growth order of $g$. Then

$$
\rho_{1} \leq\left(\frac{\rho}{\rho+1}+1\right) \rho_{2} .
$$

Proof. Again let $g$ be represented in the form $g(z)=\sum_{|\mathbf{m}|=0}^{\infty} V_{\mathbf{m}}(z) a_{\mathbf{m}}$. Applying Theorem 6 leads to

$$
\left\|a_{\mathbf{m}}\right\| \leq \exp \left(|\mathbf{m}|^{\frac{\rho}{\rho+1}+\varepsilon}\right) c(n,|\mathbf{m}|)
$$

for $\varepsilon>0$ where again $c(n,|\mathbf{m}|)=\frac{n(n+1) \cdots(n+|\mathbf{m}|-1)}{\mathbf{m} !}$. By Stirling's formula one next obtains that

$$
c(n,|\mathbf{m}|) \approx \frac{|\mathbf{m}|^{\frac{n-1}{2}} n^{\frac{n}{2}+|\mathbf{m}|}}{(n-1) !(\sqrt{2 \pi})^{n-1}} .
$$

Applying (41) and (42) leads to the following estimates

$$
\begin{aligned}
\log \left(\left\|a_{\mathbf{m}}\right\|\right) & \leq|\mathbf{m}|^{\frac{\rho}{\rho+1}+\varepsilon}+\log (c(n,|\mathbf{m}|)) \\
& \leq|\mathbf{m}|^{\frac{\rho}{\rho+1}+\varepsilon}+\frac{n-1}{2} \log (|\mathbf{m}|)+\left(\frac{n}{2}+|\mathbf{m}|\right) \log (n)+\log 2 \\
& \leq|\mathbf{m}|^{\frac{\rho}{\rho+1}+\varepsilon}+\frac{3 n-1}{2}|\mathbf{m}|+\frac{n^{2}}{2}+\log 2 \\
& \leq(n+1)|\mathbf{m}|^{\frac{\rho}{\rho+1}+\varepsilon+1}+\frac{n^{2}}{2}+\log 2
\end{aligned}
$$

In view of the definition of maximum term $\mu(r)$ of $g$ where we suppose that $r<1$ we obtain that $\mu(r)=\left\|a_{\nu(r)}\right\| r^{|\nu(r)|}$ Consequently, applying (43), we get

$$
\begin{aligned}
\log (\mu(r)) & =\log \left(\left\|a_{\nu(r)}\right\|\right)+|\nu(r)| \log (r) \\
& \leq \log \left(\left\|a_{\nu(r)}\right\|\right) \leq(n+1)|\nu(r)|^{\frac{\rho}{\rho+1}+\varepsilon+1}+\frac{n^{2}}{2}+\log 2 .
\end{aligned}
$$


Hence,

$\log \log (\mu(r))=\log (n+1)+\left(\frac{\rho}{\rho+1}+\varepsilon+1\right) \log (|\nu(r)|)+\log \left(\frac{n^{2}}{2}+\log 2\right)+\log 2$.

Finally, we obtain that

$$
\rho_{1}=\limsup _{r \rightarrow 1^{-}} \frac{\log \log (\mu(r))}{-\log (1-r)}=\left(\frac{\rho}{\rho+1}+1\right) \limsup _{r \rightarrow 1^{-}} \frac{\log (|\nu(r)|)}{-\log (1-r)} .
$$

As a further application we can establish

Theorem 9. Suppose that $g: B(0,1) \rightarrow \mathbf{C l}_{0 n}$ is a left monogenic function and that $\rho$ is the growth order of $g$. If $\rho_{2}<\infty$, then $\rho \leq 2 \rho_{2}$.

Proof. Applying Theorem 5 and Theorem 8 leads to $\rho \leq \rho_{1} \leq\left(\frac{\rho}{\rho+1}+1\right) \rho_{2}$, and $\frac{\rho}{2} \leq \rho\left(\frac{\rho+1}{2 \rho+1}\right) \leq \rho_{2}$.

\section{References}

1. M.A. Abul-Ez, D. Constales. Basic sets of polynomials in Clifford analysis. Complex Variables 14 No.1-4 (1990), 177-185.

2. M.A. Abul-Ez, D. Constales. On the order of basic series representing Clifford-valued functions. Appl. Math. Comput. 142 No. 2-3 (2003), 575584 .

3. M.A. Abul-Ez, D. Constales. On the convergence properties of basic series representing special monogenic functions. Arch. Math. 81 No.1 (2003), 6271.

4. F. Brackx, R. Delanghe and F. Sommen. Clifford Analysis. Pitman 76, London, 1982.

5. D. Constales, R. De Almeida and R. S. Kraußhar. On the relation between the growth and the Taylor coefficients of entire solutions to the higher dimensional Cauchy-Riemann system in $\mathbb{R}^{n+1}$. J. Math. Anal. Appl. 327 No. 2 (2007), 763-775.

6. D. Constales, R. De Almeida and R. S. Kraußhar. On the growth type of entire monogenic functions. Arch. Math. 88 No. 2 (2007), 153-163.

7. D. Constales and R.S. Kraußhar. Representation formulas for the general derivatives of the fundamental solution of the Cauchy-Riemann operator in Clifford Analysis and Applications. Z. Anal. Anwendungen 21 No. 3 (2002), 579-597.

8. R. De Almeida and R.S. Kraußhar. On the asymptotic growth of monogenic functions. Z. Anal. Anwendungen 24 No. 4 (2005), 791-813.

9. R. Delanghe. On the singularities of functions with values in a Clifford algebra. Math. Ann. 196 (1972), 293-319.

10. R. Fueter. Functions of a Hyper Complex Variable. Lecture notes written and supplemented by E. Bareiss, Math. Inst. Univ. Zürich, Fall Semester $1948 / 49$. 
11. K. Gürlebeck, K. Habetha, and W. Sprößig. Funktionentheorie in der Ebene und im Raum. Birkhäuser Verlag, Basel, 2006

12. K. Gürlebeck and W. Sprößig. Quaternionic and Clifford Calculus for Physicists and Engineers. John Wiley \& Sons, Chichester-New York, 1997.

13. G. Jank and L. Volkmann. Meromorphe Funktionen und Differentialgleichungen. UTB Birkhäuser, Basel 1985.

14. W. K. Hayman. The local growth of power series: a survey of the WimanValiron method. Can. Math. Bull. 17 (1974), 317-358.

15. E. Lindelöf. Sur la détermination de la croissance des fonctions entìeres définies par un développement de Taylor. Darb. Bull. 27 No.2 (1903), 213226.

16. G.R. MacLane. Asymptotic values of holomorphic functions. Rice University studies, Houston, 1963.

17. R. Nevanlinna. Zur Theorie der meromorphen Funktionen. Acta Math. 46 (1925), 1-99.

18. A. Pringsheim. Elementare Theorie der ganzen transzendenten Funktionen von endlicher Ordnung. Math. Ann. 58 (1904), 257-342.

19. M. N. Sheremeta. Maximal term and the central index of the power series expansion of a function analytic in a circle. Ukrainskii Matematicheskii Zhurnal 33 No. 6 (1981), 846-848.

20. L. R. Sons. Regularity of growth and gaps. J. Math. Anal. Appl. 24 (1968), 296-306.

21. G. Valiron. Lectures on the general theory of integral functions. Chelsea, New York, 1949.

22. A. Wiman. Über den Zusammenhang zwischen dem Maximalbetrage einer analytischen Funktion und dem größten Gliede der zugehörigen Taylorschen Reihe. Acta Math. 37 (1914), 305-326. 